ANNALES

POLONICI MATHEMATICI

$84.2(2004)$

\title{
Sur les paires d'équations pré-Schröder et leur équivalence
}

\author{
par Józef KALINOWski (Katowice)
}

\begin{abstract}
Pairs of functional pre-Schröder equations $\left(S_{n}\right)$ are considered. We show that under some assumptions the system of two equations $\left(S_{3}\right),\left(S_{n}\right)$ for some $n \geq 4$ is equivalent to the system of all equations $\left(S_{n}\right)$ for $n \geq 2$. The results answer a question of Gy. Targonski [5] in a particular case.
\end{abstract}

1. Introduction. Dans [1] et [2] on considère le problème de l'équivalence entre des équations fonctionnelles du système

$$
f^{n}(g(x))=f\left(g_{n}(x)\right) \cdot f^{n-1}(x), \quad \text { pour tout entier } n \geq 2,
$$

appelé le système d'equations pré-Schröder. Ici on emploi les notations de Z. Moszner [4] : $g$ est une application donnée, d'un ensemble $X$ en lui-même, $f: X \rightarrow Y$ une fonction inconnue, où $(Y, \cdot)$ est un demi-groupe commutatif, et $g_{n}$ pour tout entier $n \geq 0$ désigne les itérées successives de la fonction $g$, c'est-à-dire

$$
g_{0}(x)=x, \quad g_{n+1}(x)=g\left(g_{n}(x)\right) .
$$

Les équations du système $(S)$ sont désignées par $\left(S_{n}\right), n \geq 2$.

Gy. Targonski [5] a demandé si une partie du système $(S)$ est déjà équivalente à $(S)$.

2. Préliminaires. Soit $(Y, \cdot)$ un demi-groupe commutatif. Désignons par 0 un élément dans $Y$ pour lequel

$$
\bigwedge_{y \in Y} 0 \cdot y=0,
$$

si un tel élément existe. Il est évident qu'il peut en exister au plus un.

Dans ce travail nous supposerons que pour $n \in \mathbb{N}$ fixé, $n>1$, le demigroupe $(Y, \cdot)$ est sans torsion de degré $n$, c'est-à-dire

$$
\bigwedge_{x, y \in Y}\left(x^{n}=y^{n}\right) \Rightarrow(x=y) \text {. }
$$

2000 Mathematics Subject Classification: Primary 39B72.

Key words and phrases: pre-Schröder equations. 
LEMme 1 (voir [2]). Si un demi-groupe $(Y, \cdot)$ satisfait à la loi de réduction (1), alors $Y$ n'a pas de diviseurs de zéro.

Concernant l'équivalence d'une partie du système $(S)$ au tout système $(S)$, il y a des résultats suivants :

ThÉORÈme 1 (voir $[1$, théorème 1$]$ ). Soit $(Y, \cdot)$ un demi-groupe commutatif satisfaisant à la loi de réduction suivante:

$$
\bigwedge_{x, y, z \in Y}(x y=x z \wedge x \neq 0) \Rightarrow(y=z) .
$$

Si la fonction $f$ satisfait à l'équation $\left(S_{2}\right)$, alors $f$ est une solution du système $(S)$.

THÉORÈme 2 (voir [2, théorème 4$])$. Soit $(Y, \cdot)$ un demi-groupe commutatif satisfaisant à la loi de réduction (1) et sans torsion de degré $n \geq 3$. Si la fonction $f$ satisfait aux équations $\left(S_{n}\right)$ et $\left(S_{n+1}\right)$, alors $f$ est une solution du système $(S)$.

ThÉORÈme 3 (voir $[2$, théorème 5$]$ ). Soit $(Y, \cdot)$ un demi-groupe commutatif satisfaisant à la loi de réduction (1) et sans torsion de degré $n \geq 3$. Si la fonction $f$ satisfait aux équations $\left(S_{n}\right)$ et $\left(S_{2 n}\right)$, alors $f$ est une solution du système $(S)$.

THÉorème 4 (voir $[2$, théorème 6$]$ ). Soit $(Y, \cdot)$ un demi-groupe commutatif satisfaisant à la loi de réduction (1) et sans torsion de degré $n \geq 2$. Si la fonction $f$ satisfait aux équations $\left(S_{n+1}\right)$ et $\left(S_{2 n+1}\right)$, alors $f$ est une solution du système $(S)$.

Ce travail est la continuation de la recherche précédente. On va étudier la question si deux équations $\left(S_{3}\right),\left(S_{n}\right)$ pour $n \geq 4$ sont équivalentes à toutes les équations de $(S)$.

3. Lemmes auxiliaires. Maintenant nous provons quatre lemmes suivants :

Lemme 1. Soit $(Y, \cdot)$ un demi-groupe commutatif et $n \geq 4$. Si la fonction $f$ satisfait aux équations $\left(S_{3}\right)$ et $\left(S_{n}\right)$, alors

$$
f^{n}(g(x)) \cdot f^{2}\left(g_{n-3}(x)\right)=f^{3}\left(g_{n-2}(x)\right) \cdot f^{n-1}(x) .
$$

Démonstration. Soit $n \geq 4$. Multiplions par $f^{n-1}(x)$ l'équation $\left(S_{3}\right)$ avec $x$ remplacé par $g_{n-3}(x)$; en utilisant la commutativité de la multiplication, nous pouvons écrire

$$
f\left(g_{n}(x)\right) \cdot f^{n-1}(x) \cdot f^{2}\left(g_{n-3}(x)\right)=f^{3}\left(g_{n-2}(x)\right) \cdot f^{n-1}(x) .
$$

En substituant $\left(S_{n}\right)$ dans (4) nous obtenons la thèse. 
Corollaire 1. Soit $(Y, \cdot)$ un demi-groupe commutatif satisfaisant à la loi de réduction (1) et sans torsion de degré 3 . Si la fonction $f$ satisfait aux équations $\left(S_{3}\right)$ et $\left(S_{4}\right)$, alors $f$ est une solution du système $(S)$.

Démonstration. En posant $n=4$ dans l'équation (3) nous obtenons

$$
f^{6}(g(x))=f^{3}\left(g_{2}(x)\right) \cdot f^{3}(x) .
$$

Puisque $(Y, \cdot)$ est sans torsion de degré 3 , la fonction $f$ vérifie l'équation $\left(S_{2}\right)$. On peut alors appliquer le théorème 1 .

Lemme 2. Soit $(Y, \cdot)$ un demi-groupe commutatif et $n \geq 5$. Si la fonction $f$ satisfait à (3), alors

$$
f^{n}(g(x)) \cdot f^{2}\left(g_{n-3}(x)\right) \cdot f^{6}\left(g_{n-5}(x)\right)=f^{9}\left(g_{n-4}(x)\right) \cdot f^{n-1}(x) .
$$

Démonstration. En remplaçant $x$ dans $\left(S_{3}\right)$ par $g_{n-5}(x)$, puis en élévant l'équation à la puissance 3 on obtient

$$
f^{9}\left(g_{n-4}(x)\right)=f^{3}\left(g_{n-2}(x)\right) \cdot f^{6}\left(g_{n-5}(x)\right) .
$$

En multipliant (3) par $f^{6}\left(g_{n-5}(x)\right)$ et en utilisant la commutativité, nous obtenons

$$
f^{n}(g(x)) \cdot f^{2}\left(g_{n-3}(x)\right) \cdot f^{6}\left(g_{n-5}(x)\right)=f^{3}\left(g_{n-2}(x)\right) \cdot f^{6}\left(g_{n-5}(x)\right) \cdot f^{n-1}(x) .
$$

On conclut avec $(6)$.

Lemme 3. Soit $(Y, \cdot)$ un demi-groupe commutatif et $n \geq 6$. Si la fonction $f$ satisfait à (5), alors

$$
f^{n}(g(x)) \cdot f^{12}\left(g_{n-5}(x)\right)=f^{9}\left(g_{n-4}(x)\right) \cdot f^{4}\left(g_{n-6}(x)\right) \cdot f^{n-1}(x) .
$$

Démonstration. En remplaçant $x$ dans $\left(S_{3}\right)$ par $g_{n-6}(x)$ et en élévant l'équation à la puissance 2 on obtient

$$
f^{6}\left(g_{n-5}(x)\right)=f^{2}\left(g_{n-3}(x)\right) \cdot f^{4}\left(g_{n-6}(x)\right) .
$$

En multipliant (5) par $f^{4}\left(g_{n-6}(x)\right)$ et en appliquant la commutativité, nous obtenons

$$
\begin{aligned}
& f^{n}(g(x)) \cdot f^{2}\left(g_{n-3}(x)\right) \cdot f^{4}\left(g_{n-6}(x)\right) \cdot f^{6}\left(g_{n-5}(x)\right) \\
& \quad=f^{9}\left(g_{n-4}(x)\right) \cdot f^{4}\left(g_{n-6}(x)\right) \cdot f^{n-1}(x) .
\end{aligned}
$$

En utilisant (8) nous obtenons

$$
f^{n}(g(x)) \cdot f^{6}\left(g_{n-5}(x)\right) \cdot f^{6}\left(g_{n-5}(x)\right)=f^{9}\left(g_{n-4}(x)\right) \cdot f^{4}\left(g_{n-6}(x)\right) \cdot f^{n-1}(x),
$$
d'où (7).

LEMme 4. Soit $(Y, \cdot)$ un demi-groupe commutatif. Si la fonction $f$ satisfait aux équations $\left(S_{3}\right),\left(S_{n}\right)$, alors

$$
f^{n}(g(x)) \cdot f^{\alpha_{k}}\left(g_{n-2 k+1}(x)\right) \cdot f^{\beta_{k}}\left(g_{n-2 k-1}(x)\right)=f^{\gamma_{k}}\left(g_{n-2 k}(x)\right) \cdot f^{n-1}(x)
$$


pour tous nombres impairs $n, n=2 k+1, k \geq 2, k \in \mathbb{N}$, où les exposants sont donnés par

$$
\begin{aligned}
\alpha_{k} & =\frac{2}{9} \cdot 4^{k}-\frac{2}{3} \cdot k-\frac{2}{9} \\
\beta_{k} & =\frac{2}{9} \cdot 4^{k}+\frac{4}{3} \cdot k-\frac{2}{9} \\
\gamma_{k} & =\frac{4}{9} \cdot 4^{k}+\frac{2}{3} \cdot k+\frac{5}{9}
\end{aligned}
$$

ainsi que

$$
f^{n}(g(x)) \cdot f^{a_{k}}\left(g_{n-2 k-1}(x)\right)=f^{b_{k}}\left(g_{n-2 k}(x)\right) \cdot f^{c_{k}}\left(g_{n-2 k-2}(x)\right) \cdot f^{n-1}(x)
$$

pour tous nombres pairs $n, n=2 k+2, k \geq 2, k \in \mathbb{N}$, où les exposants sont donnés par

$$
\begin{aligned}
a_{k} & =\frac{8}{9} \cdot 4^{k}-\frac{2}{3} \cdot k-\frac{8}{9}, \\
b_{k} & =\frac{4}{9} \cdot 4^{k}+\frac{2}{3} \cdot k+\frac{5}{9}, \\
c_{k} & =\frac{4}{9} \cdot 4^{k}-\frac{4}{3} \cdot k-\frac{4}{9} .
\end{aligned}
$$

Démonstration. Si $f$ satisfait à $\left(S_{3}\right),\left(S_{n}\right)$, alors d'après le lemme 1 la fonction $f$ est une solution de l'équation (3). Donc en vertu des lemmes 2 et $3, f$ est une solution de (5) et (7).

Remarquons que l'équation (5) est de la forme (9) pour $k=2$, avec $\alpha_{2}=2, \beta_{2}=6, \gamma_{2}=9$. Alors les exposants $\alpha_{2}, \beta_{2}, \gamma_{2}$ sont donnés par (10)-(12) pour $k=2$.

Pareillement, l'équation (7) est de la forme (13) pour $k=2$, avec $a_{2}=12$, $b_{2}=9, c_{2}=4$. Il s'ensuit que les exposants $a_{2}, b_{2}, c_{2}$ sont donnés par (14) $-(16)$ pour $k=2$.

La preuve se fait par récurrence par rapport à $k$. Pour $k=2$ la thèse a eté démontrée ci-dessus. Supposons que les formules (9) et (13) sont vraies pour un $k \geq 2$ fixé. En remplaçant $x$ dans $\left(S_{3}\right)$ par $g_{n-2 k-2}(x)$ et en élévant l'équation à la puissance $\alpha_{k}$ on a

$$
f^{3 \alpha_{k}}\left(g_{n-2 k-1}(x)\right)=f^{\alpha_{k}}\left(g_{n-2 k+1}(x)\right) \cdot f^{2 \alpha_{k}}\left(g_{n-2 k-2}(x)\right) .
$$

En multipliant (9) par $f^{2 \alpha_{k}}\left(g_{n-2 k-2}(x)\right)$, par commutativité on obtient

$$
\begin{array}{r}
f^{n}(g(x)) \cdot f^{\alpha_{k}}\left(g_{n-2 k+1}(x)\right) \cdot f^{2 \alpha_{k}}\left(g_{n-2 k-2}(x)\right) \cdot f^{\beta_{k}}\left(g_{n-2 k-1}(x)\right) \\
=f^{\gamma_{k}}\left(g_{n-2 k}(x)\right) \cdot f^{2 \alpha_{k}}\left(g_{n-2 k-2}(x)\right) \cdot f^{n-1}(x) .
\end{array}
$$

En employant (17), on peut écrire (18) comme

$$
\begin{aligned}
f^{n}(g(x)) \cdot f^{3 \alpha_{k}+\beta_{k}} & \left(g_{n-2 k-1}(x)\right) \\
& =f^{\gamma_{k}}\left(g_{n-2 k}(x)\right) \cdot f^{2 \alpha_{k}}\left(g_{n-2 k-2}(x)\right) \cdot f^{n-1}(x) .
\end{aligned}
$$


En vertu des formules (10)-(12) et (14)-(16), on a

$$
a_{k}=3 \alpha_{k}+\beta_{k}, \quad b_{k}=\gamma_{k}, \quad c_{k}=2 \alpha_{k},
$$

ce qui donne (13) pour $k$.

En remplaçant $x$ dans $\left(S_{3}\right)$ par $g_{n-2 k-3}(x)$ et en élévant l'équation à la puissance $b_{k}$ on a

$$
f^{3 b_{k}}\left(g_{n-2 k-2}(x)\right)=f^{b_{k}}\left(g_{n-2 k}(x)\right) \cdot f^{2 b_{k}}\left(g_{n-2 k-3}(x)\right) .
$$

En multipliant (13) par $f^{2 b_{k}}\left(g_{n-2 k-3}(x)\right)$, par commutativité on obtient

$$
\begin{aligned}
& f^{n}(g(x)) \cdot f^{a_{k}}\left(g_{n-2 k-1}(x)\right) \cdot f^{2 b_{k}}\left(g_{n-2 k-3}(x)\right) \\
& \quad=f^{b_{k}}\left(g_{n-2 k}(x)\right) \cdot f^{2 b_{k}}\left(g_{n-2 k-3}(x)\right) \cdot f^{c_{k}}\left(g_{n-2 k-2}(x)\right) \cdot f^{n-1}(x) .
\end{aligned}
$$

D'après (20), l'équation (21) s'écrit

$$
\begin{aligned}
f^{n}(g(x)) \cdot f^{a_{k}}\left(g_{n-2 k-1}(x)\right) \cdot f^{2 b_{k}} & \left(g_{n-2 k-3}(x)\right) \\
& =f^{3 b_{k}+c_{k}}\left(g_{n-2 k-2}(x)\right) \cdot f^{n-1}(x) .
\end{aligned}
$$

En vertu des formules (10)-(12) et (14)-(16), on a

$$
\alpha_{k+1}=a_{k}, \quad \beta_{k+1}=2 b_{k}, \quad \gamma_{k+1}=3 b_{k}+c_{k},
$$

ce qui donne (9) pour $k+1$, c'est-à-dire

$$
\begin{aligned}
f^{n}(g(x)) \cdot f^{\alpha_{k+1}}\left(g_{n-2 k-1}(x)\right) \cdot f^{\beta_{k+1}} & \left(g_{n-2 k-3}(x)\right) \\
& =f^{\gamma_{k+1}}\left(g_{n-2 k-2}(x)\right) \cdot f^{n-1}(x) .
\end{aligned}
$$

En remplaçant $x$ dans l'équation $\left(S_{3}\right)$ par $g_{n-2 k-4}(x)$, puis après avoir élévé à la puissance $\alpha_{k+1}$ on a

$$
f^{3 \alpha_{k+1}}\left(g_{n-2 k-3}(x)\right)=f^{\alpha_{k+1}}\left(g_{n-2 k-1}(x)\right) \cdot f^{2 \alpha_{k+1}}\left(g_{n-2 k-4}(x)\right) .
$$

En multipliant (22) par $f^{2 \alpha_{k+1}}\left(g_{n-2 k-4}(x)\right)$ et par commutativité on obtient

$$
\begin{array}{r}
f^{n}(g(x)) \cdot f^{\alpha_{k+1}}\left(g_{n-2 k-1}(x)\right) \cdot f^{2 \alpha_{k+1}}\left(g_{n-2 k-4}(x)\right) \cdot f^{\beta_{k+1}}\left(g_{n-2 k-3}(x)\right) \\
=f^{\gamma_{k+1}}\left(g_{n-2 k-2}(x)\right) \cdot f^{2 \alpha_{k+1}}\left(g_{n-2 k-4}(x)\right) \cdot f^{n-1}(x) .
\end{array}
$$

En employant (24), on peut écrire (25) comme

$$
\begin{aligned}
f^{n}(g(x)) \cdot f^{3 \alpha_{k+1}+\beta_{k+1}}\left(g_{n-2 k-3}(x)\right) & \\
& =f^{\gamma_{k+1}}\left(g_{n-2 k-2}(x)\right) \cdot f^{2 \alpha_{k+1}}\left(g_{n-2 k-4}(x)\right) \cdot f^{n-1}(x) .
\end{aligned}
$$

En vertu des formules (10)-(12) et (14)-(16) on a

$$
a_{k+1}=3 \alpha_{k+1}+\beta_{k+1}, \quad b_{k+1}=\gamma_{k+1}, \quad c_{k+1}=2 \alpha_{k+1},
$$

ce qui donne (13) pour $k+1$.

Les égalités (9) et (13) sont vraies pour tout $k \geq 2$.

Remarque 1. On peut prouver par récurrence que tous les exposants $\alpha_{k}, \beta_{k}, \gamma_{k}, a_{k}, b_{k}, c_{k}$ donnés par (10)-(12) et (14)-(16) pour tout $k \geq 2$ sont des nombres naturels. 
Remarque 2. Une vérification directe montre que pour tout $k \geq 2$,

$$
\begin{gathered}
\beta_{k}=\alpha_{k}+2 k, \\
\gamma_{k}=2 \alpha_{k}+2 k+1, \\
a_{k}+1=b_{k}+c_{k}, \\
a_{k}+2 k+2=2 b_{k} .
\end{gathered}
$$

4. Le théorème principal. Maintenant nous prouvons le théorème principal de ce travail. Le théorème montre le rôle que l'équation $\left(S_{3}\right)$ joue au système des équations $(S)$.

ThÉORÈme 5. Soit $(Y, \cdot)$ un demi-groupe commutatif satisfaisant à la loi de réduction (1) et sans torsion de degré $\alpha_{k}$ donné par (10) avec $k=$ $(n-1) / 2$ pour $n \geq 4$ impair, et de degré $b_{k}$ donné par (15) avec $k=$ $(n-2) / 2$ pour $n \geq 4$ pair. Si la fonction $f$ satisfait aux équations $\left(S_{3}\right)$ et $\left(S_{n}\right)$ pour un $n \geq 4$, alors $f$ est une solution du système $(S)$.

Démonstration. Pour $n=4$ la thèse résulte du corollaire 1.

Posons $n=2 k+1$ avec $k \geq 2$. On emploie le lemme 4. D'après (9) nous obtenons

$$
f^{2 k+1}(g(x)) \cdot f^{\alpha_{k}}\left(g_{2}(x)\right) \cdot f^{\beta_{k}}(x)=f^{\gamma_{k}}(g(x)) \cdot f^{2 k}(x) .
$$

Des égalités (26), (27) il suit que

$$
f^{2 k+1}(g(x)) \cdot f^{\alpha_{k}}\left(g_{2}(x)\right) \cdot f^{\alpha_{k}+2 k}(x)=f^{2 \alpha_{k}+2 k+1}(g(x)) \cdot f^{2 k}(x) .
$$

Si $f(g(x)) \neq 0$ la loi de réduction (1) donne

$$
f^{\alpha_{k}}\left(g_{2}(x)\right) \cdot f^{\alpha_{k}+2 k}(x)=f^{2 \alpha_{k}}(g(x)) \cdot f^{2 k}(x) .
$$

De $f(g(x)) \neq 0$ en employant $\left(S_{3}\right)$ nous obtenons $f(x) \neq 0$. La loi de réduction (1) et la commutativité nous donnent encore

$$
\left[f\left(g_{2}(x)\right) \cdot f(x)\right]^{\alpha_{k}}=\left[f^{2}(g(x))\right]^{\alpha_{k}} .
$$

Si $f(g(x))=0$, de $\left(S_{3}\right)$ avec $x$ remplacé par $g(x)$, il vient que $f\left(g_{2}(x)\right)=0$ et on déduit (31) aussi.

Puisque $(Y, \cdot)$ est un demi-groupe sans torsion de degré $\alpha_{k}$ pour $k=$ $(n-1) / 2$, la fonction $f$ vérifie $\left(S_{2}\right)$. On peut alors appliquer le théorème 1 et on déduit la thèse pour tous $n$ impair, $n \geq 5$.

Posons $n=2 k+2$ pour $k \geq 2$. On emploi le lemme 4 . D'après (13) nous obtenons

$$
f^{2 k+2}(g(x)) \cdot f^{a_{k}}(g(x))=f^{b_{k}}\left(g_{2}(x)\right) \cdot f^{c_{k}}(x) \cdot f^{2 k+1}(x) .
$$

Des égalités (15), (16) il suit que $b_{k}=c_{k}+2 k+1$, donc

$$
f^{2 k+2}(g(x)) \cdot f^{a_{k}}(g(x))=f^{b_{k}}\left(g_{2}(x)\right) \cdot f^{b_{k}}(x) .
$$


De l'égalité (29) il vient que $a_{k}=2 b_{k}-2 k-2$. Par commutativité nous obtenons

$$
\left[f^{2}(g(x))\right]^{b_{k}}=\left[f\left(g_{2}(x)\right) \cdot f(x)\right]^{b_{k}} .
$$

Puisque $(Y, \cdot)$ est un demi-groupe sans torsion de degré $b_{k}$ pour $k=(n-2) / 2$, la fonction $f$ vérifie $\left(S_{2}\right)$. On peut alors appliquer le théorème 1 et on déduit la thèse pour tous $n$ pair, $n \geq 6$.

De la considération précédente on obtient que les équations $\left(S_{3}\right),\left(S_{n}\right)$ pour $n \geq 4$ sont équivalentes au système $(S)$. Ainsi, le théorème est démontré.

Remarque 3. Il résulte du travail [2] que le système $(S)$ est équivalent à chaque des systèmes $\left(S_{3}\right),\left(S_{4}\right)$ (théorème 2$),\left(S_{3}\right),\left(S_{5}\right)$ (théorème 4 ) et $\left(S_{3}\right)$, ( $\left.S_{6}\right)$ (théorème 3$)$.

ExEmple 1. Soit $Z$ un ensemble infini, $g: Z \rightarrow Z$ une fonction. Si $x_{0} \in Z$, nous définissons

$$
x_{n}=g_{n}\left(x_{0}\right) \quad \text { pour } n \in \mathbb{N} \cup\{0\} .
$$

Supposons que

$$
x_{n} \neq x_{m} \quad \text { pour } n \neq m \text {. }
$$

Soit $X=\left\{x_{0}, x_{1}, x_{2}, \ldots\right\}$. L'ensemble $Y=\{-1,1\}$ avec multiplication est un groupe commutatif avec la loi de réduction $(1)$. La structure $(Y, \cdot)$ est sans torsion de degré pair.

La fonction

$$
f\left(x_{n}\right)= \begin{cases}-1 & \text { pour } n=0 \\ 1 & \text { pour } n \in \mathbb{N}\end{cases}
$$

est une solution des équations $\left(S_{3}\right)$, $\left(S_{2 n+1}\right)$ pour tous $n \geq 2$, dans l'ensemble $X$.

En effet, pour $x_{0} \in X$ on a

$$
\begin{gathered}
1=1^{3}=f^{3}\left(g\left(x_{0}\right)\right)=f\left(g_{3}\left(x_{0}\right)\right) \cdot f^{2}\left(x_{0}\right)=1 \cdot(-1)^{2}=1 \\
1=1^{2 n+1}=f^{2 n+1}\left(g\left(x_{0}\right)\right)=f\left(g_{2 n+1}\left(x_{0}\right)\right) \cdot f^{2 n}\left(x_{0}\right)=1 \cdot(-1)^{2 n}=1 .
\end{gathered}
$$

Pour $x_{m} \in X, m \in \mathbb{N}$ nous obtenons

$$
\begin{gathered}
1=1^{3}=f^{3}\left(g\left(x_{m}\right)\right)=f\left(g_{3}\left(x_{m}\right)\right) \cdot f^{2}\left(x_{m}\right)=1 \cdot 1^{2}=1 \\
1=1^{2 n+1}=f^{2 n+1}\left(g\left(x_{m}\right)\right)=f\left(g_{2 n+1}\left(x_{m}\right)\right) \cdot f^{2 n}\left(x_{m}\right)=1 \cdot 1^{2 n}=1 .
\end{gathered}
$$

Nous observons que la fonction $f$ ne vérifie pas l'équation $\left(S_{2}\right)$ dans $X$, parce que pour $x_{0} \in X$,

$$
1=1^{2}=f^{2}\left(g\left(x_{0}\right)\right) \neq f\left(g_{2}\left(x_{0}\right)\right) \cdot f^{1}\left(x_{0}\right)=1 \cdot(-1)^{1}=-1 .
$$

Donc, les hyphothèses que l'ensemble $Y$ est un demi-groupe commutatif avec la loi de réduction (1) ne suffit pas pour que le théorème 5 soit vrai. Il faut supposer que la structure $(Y, \cdot)$ est sans torsion. 


\section{Références}

[1] J. Drewniak et J. Kalinowski, Les relations entre les équations pré-Schröder I, Ann. Polon. Math. 32 (1976), 5-11.

[2] J. Kalinowski, L'équivalence entre les équations pré-Schröder, en préparation.

[3] M. Kuczma, B. Choczewski and R. Ger, Iterative Functional Equations, Encyclopedia Math. Appl. 32, Cambridge Univ. Press, 1990.

[4] Z. Moszner, Sur un problème relatif aux équations de pré-Schröder, Ann. Polon. Math. 27 (1973), 289-292.

[5] Gy. Targonski, Problem P 63, Aequationes Math. 4 (1970), 251.

Department of Mathematics

Silesian University

Bankowa 14

40-007 Katowice, Poland

E-mail: kalinows@ux2.math.us.edu.pl

Reçu par la Rédaction le 19.5.2004

Révisé le 5.11.2004 